\title{
Derivation of asymptotical formulas for resolution of systems of differential equations with symmetrical matrices
}

\author{
M.I. SHKIL', P.F. SAMUSENKO \\ Drahomanov Pedagogical University, \\ 9 Pyrogov Street, Kyïv, Ukraïna
}

\begin{abstract}
Asymptotic formulae for resolution of $L$-diagonal systems of ordinary differential equations with symmetrical matrices are derived.
\end{abstract}

1) We shall consider a system of linear differential equations

$$
\frac{d x}{d t}=(\Lambda(t)+Q(t)) x
$$

where $x$ is an $n$-vector, $\Lambda(t)$ is an $(n \times n)$ diagonal matrix, and $Q(t)$ is an $(n \times n)$-matrix with summable elements in the interval $\left(t_{0}, \infty\right)$. Such a system was called $L$-diagonal system by I.M. Rapoport [1]. Studying these systems we assume that:

a) The elements $\omega_{i}(t) \quad(i=1,2, \ldots, n)$ of the diagonal matrix $\Lambda(t)$ are summable in the interval $\left(t_{0}, t_{1}\right)$ for any finite $t_{1}$;

b) There exists $T_{0}$ big enough for any difference

$$
\operatorname{Re} \omega_{i}(t)-\operatorname{Re} \omega_{j}(t), i, j=1, \ldots, n,
$$

not to change sign for $t \geq T_{0}$.

Then system (1) can be solved for $t \geq t_{0}$ and its $n$ particular solutions have the form

$$
x_{i}=\eta_{i j}(t) \exp \int_{t_{0}}^{t} \omega_{j}(t) d t, \quad i, j=1,2, \ldots, n,
$$

where $\eta_{i j}(t)$ are continuous functions in the closed interval $\left[t_{0}, \infty\right]$, and $\eta_{i j}(\infty)=0$ when $i \neq j, \eta_{j j}(\infty)=1$. I.M. Rapoport [1] found substitutions which can help us to reduce systems of differential equations

$$
\frac{d x}{d t}=A(t) x
$$

to $L$-diagonal systems in the case when roots of the characteristic equation are simple. In the paper [2] we suggested the method for construction of the mentioned substitutions in the case when the roots of the characteristic equation for $t \geq t_{0}$ maintain constant multiplicity. 
2) In this paper we suggest the method for construction of such substitutions for systems of differential equations

$$
B(t) \frac{d x}{d t}=A(t) x
$$

where the matrix $B(t)$ may not have an inverse matrix, when $t \geq t_{0}$.

3) We will consider instead of the system (3) the system

$$
\varepsilon B(t) \frac{d x}{d t}=A(t) x
$$

where $\varepsilon>0$ is a real parameter.

The system $\left(3^{\prime}\right)$ coincides with the system (3) when $\varepsilon=1$. We shall make use of a substitution $t=\varepsilon t_{1}$ in the system $\left(3^{\prime}\right)$. Now we have the system

$$
B(t) \frac{d x}{d t_{1}}=A(t) x
$$

For construction of a fundamental matrix of solutions for this system, we can use the method from [2].

The equation

$$
\operatorname{det}(A(t)-\lambda B(t))=0
$$

has $v$ roots $\lambda(t), \ldots, \lambda_{v}(t) \quad(v \leq n)$.

We assume that the matrices $A(t), B(t)$ are symmetric for $t \geq t_{0}$. Then the roots of the equation (5) are real [3]. We assume that the roots $\lambda_{i}(t)$ are different when $t \leq t<\infty$. Hence when $t \geq t_{0} \quad \lambda_{i}(t) \neq \lambda_{j}(t), \quad i \neq j, \quad i, j=1, \ldots, v$, so we can construct proper vectors $\mu_{i}(t)$ of the matrix $A(t)$ with respect to the matrix $B(t)$ in order for the scalar product

$$
\left(B(t) \mu_{i}(t), \mu_{j}(t)\right)= \begin{cases}1, & i=j \\ 0, & i \neq j, \quad i, j=1, \ldots, v .\end{cases}
$$

We put

$$
x=\mathcal{U}_{m}(t, \varepsilon) y, \quad \mathcal{U}_{m}(t, \varepsilon)=\sum_{s=0}^{m} \varepsilon^{s} \mathcal{U}_{s}(t),
$$

where $y$ is an $n$-measurable vector, and $\mathcal{U}_{s}(t)$ are square $(n \times n)$-matrices. We have

$$
B(t) \mathcal{U}_{m}(t, \varepsilon) \frac{d y}{d t_{1}}=\left(A(t) \mathcal{U}_{m}(t, \varepsilon)-\varepsilon B(t) \mathcal{U}_{m}^{\prime}(t, \varepsilon)\right) y
$$

We construct the matrices $\mathcal{U}_{s}(t) \quad(s=0,1, \ldots, m)$ so as to get the matrix equality

$$
A(t) \mathcal{U}_{m}(t, \varepsilon)-\varepsilon B(t) \mathcal{U}_{m}^{\prime}(t, \varepsilon)=B(t) \mathcal{U}_{m}(t, \varepsilon)\left(\Lambda(t, \varepsilon)+\varepsilon^{m+1} C_{m}(t, \varepsilon)\right),
$$

where $\Lambda(t, \varepsilon)$ is a diagonal matrix,

$$
\Lambda_{m}(t, \varepsilon)=\sum_{s=0}^{m} \varepsilon^{s} \Lambda_{s}(t)
$$


We have to compare coefficients of $\varepsilon^{0}, \varepsilon^{1}, \ldots, \varepsilon^{m}$ in the matrix equalities (6). We have a matrix system of equations

$$
\begin{aligned}
& A(t) \mathcal{U}_{0}(t)-B(t) \mathcal{U}_{0}(t) \Lambda_{0}(t)=0 \\
& A(t) \mathcal{U}_{s}(t)-B(t) \mathcal{U}_{s}(t) \Lambda_{0}(t)=B(t) \mathcal{U}_{s-1}^{\prime}(t)+B(t) \sum_{j=1}^{s} \mathcal{U}_{s-j}(t) \Lambda_{j}(t)
\end{aligned}
$$

Let $\Lambda_{0}(t)=\operatorname{diag}\left\{\lambda-1(t), \lambda_{2}(t), \ldots, \lambda_{v}(t), \ldots, \lambda_{n}(t)\right\}$.

We write the matrix equation (7) in the vector form and designate columns of the matrix $\mathcal{U}_{0}(t)$ by $u_{0 i}(t) \quad(i=1,2, \ldots, n)$. We have

$$
\left(A(t)-\lambda_{j}(t) B(t)\right) u_{0 i}(t)=0 .
$$

Then

$$
u_{o i}(t)=\mu_{i}(t) \quad(i=1,2, \ldots, n) \text { and } u_{0 i}(t) \equiv 0 \quad(i=v+1, \ldots, n) .
$$

Let us consider (8) when $s=1$ :

$$
A(t) \mathcal{U}_{1}(t)-B(t) \mathcal{U}_{1}(t) \Lambda_{0}(t)=B(t) \mathcal{U}_{0}^{\prime}(t)+B(t) \mathcal{U}_{0}(t) \Lambda_{1}(t)
$$

or in the vector form

$$
\left(A(t)-\lambda_{i}(t) B(t)\right) u_{1 i}(t)=B(t) u_{0 i}^{\prime}(t)+B(t) u_{0 i}(t) \lambda_{1 i}(t) \equiv g_{1 i}(t), \quad i=1,2, \ldots, n .
$$

The equation (9) can be solved relatively $u_{1 i}(t)$ iff, when the vector $g_{1 i}(t) \quad(i=1,2, \ldots, n)$ is orthogonal to the vector which is a solution of the conjugated system corresponding to the homogeneous system $(9) . A(t)$ and $B(t)$ are symmetric, so the conjugated system coincides with (9). So (9) has a solution iff, when

$$
\left(g_{1 i}(t), \mu_{i}(t)\right)=0
$$

for all $t \geq t_{0}$. For $i=1, \ldots, n$ we have

$$
\left(B(t) u_{0 i}^{\prime}(t), \mu_{i}(t)\right)+\left(B(t) u_{0 i}(t) \lambda_{1 i}(t), \mu_{i}(t)\right)=0
$$

or

$$
\lambda_{1 i}(t)=-\left(B(t) u_{o i}^{\prime}(t), \mu_{i}(t)\right), \quad i=1,2, \ldots, n .
$$

If $i=v+1, \ldots, n$, then (10) will change to an identity. So we can take $\lambda_{1 i}(t) \equiv 0 \quad(i=$ $v+1, \ldots, n)$. Thus substituting the obtained values of $\lambda_{1 i}(t) \quad(i=1, \ldots, n)$ to the system (9), we get the vector-column of the first part is orthogonal for all $t \geq t_{0}$ to a non-trivial solution of the conjugate system. We look for this solution in such a form

$$
u_{1 i}(t)=\sum_{r=1}^{v} c_{r i}^{(1)}(t) \mu_{r}(t), \quad i=1, \ldots, v
$$

where $c_{r i}^{(1)}(t)$ is a function which must be defined for the vector (11) to satisfy the system $(9)$. 
Substituting (11) to system (9) and multiplying this result by the vector $\mu_{j}(t) \quad(j=$ $1, \ldots, v)$, we have

$$
c_{j i}^{(1)}(t)\left(\lambda_{j}(t)-\lambda_{i}(t)\right)=\left(g_{1 i}(t), \mu_{j}(t)\right), \quad j=1, \ldots, v .
$$

When $i=j$ we have the identity $c_{j j}^{(1)}(t) \cdot 0 \equiv 0$. Hence we can take any function $c_{j j}^{(1)}(t)$. We take $c_{j j}^{(1)}(t) \equiv 0$, for $t \geq t_{0}$. When $i \neq j$

$$
c_{j i}^{(1)}(t)=\frac{\left(g_{1 i}(t), \mu_{j}(t)\right)}{\lambda_{j}(t)-\lambda_{i}(t)} .
$$

Then

$$
u_{1 i}(t)=\sum_{r=1}^{v} \frac{\left(g_{1 i}(t), \mu_{j}(t)\right)}{\lambda_{j}(t)-\lambda_{i}(t)} \mu_{r}(t) .
$$

We assume that $u_{1 i}(t) \equiv 0, \quad i=v+1, \ldots, n$.

Thus we defined the vectors $u_{1 i}(t) \quad(i=1,2, \ldots, n)$ (in the matrix $\left.\mathcal{U}_{1}(t)\right)$ and functions $\lambda_{1 i}(t) \quad(i=1,2, \ldots, n)$ (in the matrix $\left.\Lambda_{1}(t)\right)$. Using the method of mathematical induction, we can find from equations (8) all the following matrices $\mathcal{U}_{s}(t)$ and $\Lambda_{s}(t) \quad(s=2,3, \ldots, m)$. So the system (4) has the form

$$
B(t) \mathcal{U}_{m}(t, \varepsilon) \frac{d y}{d t_{1}}=B(t) \mathcal{U}_{m}(t, \varepsilon)\left(\Lambda_{m}(t, \varepsilon)+\varepsilon^{m+1} C_{m}(t, \varepsilon)\right) y .
$$

We can find the matrix $C_{m}(t, \varepsilon)$ from (6)

$$
\varepsilon^{m+1} B(t) \mathcal{U}_{m}(t, \varepsilon) C_{m}(t, \varepsilon)=A(t) \mathcal{U}_{m}(t, \varepsilon)-\varepsilon B(t) \mathcal{U}_{m}^{\prime}(t, \varepsilon)-B(t) \mathcal{U}_{m}(t, \varepsilon) \Lambda_{m}(t, \varepsilon) .
$$

We assume that

$$
\left(E-B(t) \mathcal{U}_{m}(t, 1)\left(B(t) \mathcal{U}_{m}(t, 1)\right)^{-}\right) D_{m}(t, 1)=0,
$$

is a true equality for $\varepsilon=1$ and $t_{0} \leq t<+\infty$, where $\left(B(t) \mathcal{U}_{m}(t, 1)\right)^{-}$is a half-inverse matrix for the matrix $B(t) \mathcal{U}_{m}(t, 1)$,

$$
D_{m}(t, 1)=-B(t)\left(\mathcal{U}_{m}^{\prime}(t, 1)+\sum_{r=1}^{m} \sum_{j=r}^{m} \mathcal{U}_{j}(t) \Lambda_{m+r-j}(t)\right) .
$$

Then

$$
C_{m}(t, 1)=\left(B(t) \mathcal{U}_{m}(t, 1)\right)^{-} D_{m}(t, 1) .
$$

So, we have

$$
B(t) \mathcal{U}_{m}(t, 1) \frac{d y}{d t}=B(t) \mathcal{U}_{m}(t, 1)\left(\Lambda_{m}(t, 1)+C_{m}(t, 1)\right) y .
$$

Let the system

$$
\frac{d y}{d t}=\left(\Lambda_{m}(t, 1)+C_{m}(t, 1)\right) y, \quad t \geq t_{0}
$$


be L-diagonalizable. We can understand that every solution of (13) is a solution of (12). With the condition $x=\mathcal{U}_{m}(t, 1) y$ we can find solutions of the system (3). Looking at the conditions for matrices $A(t), B(t)$, when $t \geq t_{0}$, we receive solutions of (3) in such a form

$$
x_{j}=\mu_{i j}(t) \exp \int_{t_{0}}^{t} \omega_{j}(t) d t, \quad i, j=1,2, \ldots, n,
$$

where $\mu_{i j}(t)$ are continuous functions on the interval $\left[t_{0}, \infty\right)$.

Theorem 1. For the system (3) the following is true:

1) matrices $A(t)$ and $B(t)$ on the interval $\left[t_{0}, \infty\right)$ have continuous derivatives;

2) $A(t), B(t)$ are symmetric when $t \geq t_{0}$;

3) roots $\lambda_{i}(t)(i=1,2, \ldots, v)$ of the equation

$$
\operatorname{det}(A(t)-\lambda B(t))=0
$$

when $t \geq t_{0}$, are simple;

4) when $\varepsilon=1$ and $t_{0} \leq t<+\infty$ the equality

$$
\left(E-B(t) \mathcal{U}_{m}(t, 1)\left(B(t) \mathcal{U}_{m}(t, 1)\right)^{-}\right) D_{m}(t, 1)=0,
$$

holds, where $\mathcal{U}_{m}(t, 1), D_{m}(t, 1)$ are the matrices that we have found.

If the system (13) is a $L$-diagonal system, then $n$ particular solutions of system (3) have the form

$$
x_{i}=\mu_{i j}(t) \exp \int_{t_{0}}^{t} \omega_{j}(t) d t, \quad i, j=1,2, \ldots, n,
$$

where $\mu_{i j}(t)$ are continuous functions on the interval $\left[t_{0}, \infty\right)$.

\section{References}

[1] Rapoport I.M., On Some Asymptotic Methods in Theory of Differential Equations, Kiev, Vydavnytstvo AN UkSSR, 1954, 290p.

[2] Shkil' M.I., Asymptotic Methods in Differential Equations, Kiev, Vyshcha shkola, 1971, 226p.

[3] Gantmakher F.R., Theory of Matrices, Moskow, Nauka, 1988, 548p.

[4] Shkil' M.I., Starun I.I., Yacovec V.P., Asymptotic Integration of Linear Systems of Differential Equations, Kiev, Vyshcha shkola, 1991, 207p. 